\title{
FAKE BOUNDARY SETS IN THE HILBERT CUBE
}

\author{
PHILIP L. BOWERS
}

\begin{abstract}
For each positive integer $n$, a $\sigma$ - $Z$-set $B_{n}$ in the Hilbert cube $I^{\infty}$ is constructed whose complement $s_{n}=I^{\infty}-B_{n}$ is not homeomorphic to the pseudointerior $s$ of the Hilbert cube though $s_{n}$ and $B_{n}$ satisfy: (i) every compact subset of $s_{n}$ is a $Z$-set in $s_{n}$; (ii) $s_{n} \times s_{n}$ is homeomorphic to $s$; (iii) $B_{n}$ admits small maps $I^{\infty} \rightarrow B_{n}$; (iv) $s_{n}$ satisfies the discrete $n$-cells property; and (v) $B_{n}$ is locally $(n-1)$ connected in $I^{\infty}$. It is shown that $s_{n}$ does not satisfy the discrete $(n+1)$-cells property and thus $B_{n}$ is not a boundary set, that is, $s_{n}$ is not homeomorphic to $s$. These examples build upon an example of Anderson, Curtis, and van Mill of a fake boundary set $B_{0}$ that satisfies (i)-(iv) for $n=0$. Their example is not a boundary set since it fails to be locally continuum-connected. The examples constructed herein show that there is a hierarchy of fake boundary sets satisfying (i)-(iv) that satisfy higher and higher orders of a strong form of local connectivity (v).
\end{abstract}

1. Introduction. A boundary set in the Hilbert cube $I^{\infty}$ is a $\sigma$-Z-set $B \subset I^{\infty}$ for which $I^{\infty}-B$ is homeomorphic to the pseudo-interior $s$ of the Hilbert cube. In [5] Curtis introduces the notion of boundary sets and gives various necessary and sufficient conditions for a $\sigma$-Z-set in the Hilbert cube to be a boundary set. One of Curtis' characterizations of boundary sets takes the following form:

TheOREM 1.1. $A$ dense $\sigma-Z$-set $B \subset I^{\infty}$ is a boundary set if and only if: for every $\varepsilon>0$ there exists a $\delta>0$ such that, for every compact set $S \subset B$ with $\operatorname{diam} S<\delta$, there exists a compact set $K \subset B$ with $\operatorname{diam} K<\varepsilon$ such that $S$ contracts to a point in every neighborhood of $K$ in $I^{\infty}$.

One consequence of this theorem is that every boundary set must be locally connected in each dimension in a continuum sense that is made precise in \$2. In particular, every boundary set is locally continuum-connected.

Recognizing boundary sets can be a rather subtle problem, as evidenced by the following example of Anderson, Curtis and van Mill [1].

EXAMPLE 1.2. There exists a $\sigma$-Z-set $B_{0} \subset I^{\infty}$ that is not a boundary set though it satisfies:

(i) $I^{\infty}-B_{0}$ is an infinite-dimensional topologically complete separable $A R$ that embeds as a convex subset of $l_{2}$;

Received by the editors January 6, 1984 and, in revised form, March 30, 1984.

1980 Mathematics Subject Classification. Primary 57N20; Secondary 54F35.

Key words and phrases. Boundary set in the Hilbert cube, discrete approximation property, discrete $n$-cells property, locally $n$-connected in $X$. 
(ii) every compact subset of $I^{\infty}-B_{0}$ is a Z-set;

(iii) $\left(I^{\infty}-B_{0}\right) \times\left(I^{\infty}-B_{0}\right)$ is homeomorphic to $s$;

(iv) $B_{0}$ admits small maps $I^{\infty} \rightarrow B_{0}$; that is, for every $\varepsilon>0$, the identity map on $I^{\infty}$ is $\varepsilon$-close to a map on $I^{\infty}$ whose image lies in $B_{0}$.

The "fake" boundary set $B_{0}$ is not a boundary set since it fails to be locally continuum-connected. In [6] Curtis asks whether or not a locally continuum-connected $\sigma$ - $Z$-set $B$ in the Hilbert cube that admits small maps $I^{\infty} \rightarrow B$ is a boundary set.

In this paper we produce a hierarchy of examples of fake boundary sets that satisfy higher and higher orders of local connectivity. Specifically, for each positive integer $n$, we produce a $\sigma$-Z-set $B_{n}$ in the Hilbert cube that satisfies (i)-(iv) of Example 1.2 and is locally $(n-1)$-connected in $I^{\infty}$, but not locally $n$-connected in $I^{\infty}$. See Definition 2.1. In fact, $I^{\infty}-B_{n}$ satisfies the $n$-dimensional version, but not the $(n+1)$-dimensional version, of the discrete approximation property that Torunczyk shows characterizes those topologically complete AR's that are homeomorphic to the pseudo-interior of the Hilbert cube [10]. Notice that each of our examples provides a negative answer to Curtis' question that is mentioned in the preceding paragraph.

The paper is organized as follows. In $\S 2$, for each nonnegative integer $n$, we define two versions of what it means for a subset of a space to be locally $n$-connected in that space. We then define the discrete $n$-cells property, the $n$-dimensional version of the discrete approximation property of Torunczyk, and we state the relationship between this and the two previous definitions. In particular, we state that if a dense $\sigma$ - $Z$-set $F$ in a locally compact separable metric space $X$ is $\mathrm{LC}^{n-1}$ in $X$, then $X-F$ satisfies the discrete $n$-cells property. This allows us to conclude that the complement in the Hilbert cube of the fake boundary set $B_{n}$ satisfies the discrete $n$-cells property. In $\$ 3$ we construct our examples and show that the $n$th example, $B_{n}$, is $\mathrm{LC}^{n-1}$ in $I^{\infty}$. We show that $B_{n}$ is not a boundary set by showing that $B_{n}$ is not proximately $\mathrm{LC}^{n}$ in $I^{\infty}$, which every boundary set must be.

The reader who is familiar with the notion of boundary sets and their characterizations may wish to proceed directly to the construction of our examples in $\S 3$.

Notation. By $I$ we denote the interval $[-1,1]$, by $\mathbb{N}$ the collection of positive integers; continuous functions are called "maps". All spaces are assumed to be separable and metrizable, and if $X$ is a space, then $\rho$ usually denotes any metric on $X$ compatible with the topology on $X$. For $n \in \mathbb{N}, I^{n}$ denotes the standard $n$-cell, the $n$-fold product of $I$ with itself, $S^{n}$ denotes the standard $n$-sphere, $I^{\infty}=\prod_{n \in N}[-1,1]_{n}$ denotes the Hilbert cube, and $s=\prod_{n \in N}(-1,1)_{n}$ its pseudo-interior. If $f$ and $g$ are maps of a space $X$ into a space $Y, \mathscr{U}$ is an open cover of $Y$, and $\varepsilon: Y \rightarrow(0, \infty)$ is a map, then $f$ is said to be $\mathscr{U}$-close (respectivel $;, \varepsilon$-close) to $g$ provided for every $x \in X$, $\{f(x), g(x)\} \subset U$ for some $U$ in $\mathscr{U}$ (respectively, for every $x \in X$, $\rho(f(x), g(x))<\varepsilon(f(x)))$. $X \approx Y$ means $X$ is homeomorphic to $Y$. By $i, k, n$, we denote elements of $\mathbb{N} \cup\{0\} \cup\{\infty\}$, and $i<n$ means " $i \leqslant n-1$ if $n \neq \infty$ and $i \neq \infty$ if $n=\infty "$, and $\infty+1$ and $\infty-1$ mean $\infty$. 
2. Preliminaries. Throughout this section we fix an element $n$ of $\mathbb{N} \cup\{0\} \cup\{\infty\}$.

Definition 2.1. For $n<\infty$, a subset $F$ of a space $X$ is locally $n$-connected in $X$ provided for every $x \in X$ and neighborhood $U$ of $x$ in $X$, there exists a neighborhood $V$ of $x$ in $X$ such that every map $f: S^{n} \rightarrow V \cap F$ is null-homotopic in $U \cap F$. We say that $F$ is $\mathrm{LC}^{n}$ in $X$ if it is locally $i$-connected in $X$ for $0 \leqslant i \leqslant n$, and $F$ is LC $^{\infty}$ in $X$ if it is locally $i$-connected in $X$ for all $i \geqslant 0$.

The property of being $\mathrm{LC}^{n}$ in $X$ is not an intrinsic property of the subset $F$ of $X$. Rather, this property combines an intrinsic property of $F$ with a property of the particular embedding of $F$ into $X$. Indeed, $F$ is $\mathrm{LC}^{n}$ in $X$ if and only if $F$ is an $\mathrm{LC}^{n}$ space and $X-F$ is an $\mathrm{LCC}^{n}$ subset of $X$, the former property being an intrinsic property of $F$ and the latter a property of the embedding of $F$ into $X$. For example, $(0,1) \approx\left\{e^{i t} \in S^{1} \mid 0<t<1\right\}$ is $\operatorname{LC}^{0}$ in $S^{1}$, while $(0,1) \approx\left\{e^{2 \pi i t} \in S^{1} \mid 0<t<1\right\}$ is not $\mathrm{LC}^{0}$ in $S^{1}$.

We now define a continuum version of local connectivity in a space. This definition reflects the fact that a subset $F$ of a space $X$ may fail to have nice local connectivity properties, yet it may be embedded in the overlying space $X$ in such a way that nice local connectivity properties abound for open neighborhoods of $F$. This definition is inspired by Curtis [5] and arises naturally in the study of discrete cells properties (see [2, 3, and 5]).

Definition 2.2. For $n<\infty$, a subset $F$ of a space $X$ is proximately locally $n$-connected in $X$ provided for every $x \in X$ and neighborhood $U$ of $x$ in $X$, there exists a neighborhood $V$ of $x$ in $X$ such that, for every compactum $S \subset V \cap F$, there exists a compactum $K \subset U \cap F$ such that, for every neighborhood $N(K)$ of $K$ in $X$, there exists a neighborhood $N(S)$ of $S$ in $X$ such that every map $f: S^{n} \rightarrow N(S)$ is null-homotopic in $N(K)$. We say that $F$ is proximately $\mathrm{LC}^{n}$ in $X$ if it is proximately locally $i$-connected in $X$ for $0 \leqslant i \leqslant n$, and $F$ is proximately $\mathrm{LC}^{\infty}$ in $X$ if it is proximately locally $i$-connected in $X$ for all $i \geqslant 0$.

If we require $X$ to be an ANR and replace "for every $x \in X$ " by "for every $x \in F$ " in Definition 2.2, we arrive at Curtis' definition of proximately locally $n$-connected, proximately $\mathrm{LC}^{n}$, and proximately $\mathrm{LC}^{\infty}$. These properties are intrinsic properties of $F$, independent of the particular embedding of $F$ into any ANR. The property of being proximately $\mathrm{LC}^{n}$ in $X$ holds the same relationship to being proximately $\mathrm{LC}^{n}$ as the property of being $\mathrm{LC}^{n}$ in $X$ holds to being $\mathrm{LC}^{n}$. Indeed, $F$ is proximately $\mathrm{LC}^{n}$ in $X$ if and only if $F$ is a proximately $\mathrm{LC}^{n}$, an intrinsic property of $F$, and $F$ is an $(n+1)$-target dense embedding in $X$ (maps of $(n+1)$-cells into $X$ can be pushed close to $F$ by small moves). See Curtis [5].

We are ready to define various discrete cells properties and to state the relationships between these and the two preceding versions of local connectivity in a space. Torunczyk [10] has obtained the following topological characterization of the pseudo-interior $s$ of the Hilbert cube:

THEOREM 2.3. A topologically complete separable AR $X$ is homeomorphic to $s$ if and only if every map $f: \bigoplus_{i \in \mathbf{N}} I_{i}^{\infty} \rightarrow X$ of the countable free union of Hilbert cubes into $X$ is strongly approximable by maps $g: \bigoplus_{i \in \mathrm{N}} I_{i}^{\infty} \rightarrow X$ for which the collection $\left\{g\left(I_{i}^{\infty}\right)\right\}_{i \in \mathrm{N}}$ is discrete. 
The above approximation property is referred to as the strong discrete approximation property in [1]; however, we shall delete the adjective strong and refer to this as the discrete approximation property. Recall that a collection $\mathscr{D}$ of subsets of $X$ is discrete provided every point in $X$ has a neighborhood that meets at most one element of $\mathscr{D}$. If we replace the countable free union of Hilbert cubes by the countable free union of $n$-cells, we obtain a hierarchy of discrete cells properties:

Definition 2.4. A metric space $X$ satisfies the discrete $n$-cells property (discrete $\infty$-cells property $=$ discrete approximation property) provided either of the following equivalent conditions is satisfied:

2.4.1. For each map $f: \bigoplus_{i \in N} I_{i}^{n} \rightarrow X$ and each open cover $\mathscr{U}$ of $X$, there exists a map $g: \bigoplus_{i \in N} I_{i}^{n} \rightarrow X$ such that $f$ and $g$ are $\mathscr{U}$-close and $\left\{g\left(I_{i}^{n}\right)\right\}_{i \in \mathrm{N}}$ is discrete.

2.4.2. For each map $f: \bigoplus_{i \in N} I_{i}^{n} \rightarrow X$ and each map $\varepsilon: X \rightarrow(0, \infty)$, there exists a map $g: \bigoplus_{i \in \mathbf{N}} I_{i}^{n} \rightarrow X$ such that $f$ and $g$ are $\varepsilon$-close and $\left\{g\left(I_{i}^{n}\right)\right\}_{i \in \mathbf{N}}$ is discrete.

THEOREM 2.5. A topologically complete separable ANR X satisfies the discrete $n$-cells property if and only if every map from any topologically complete separable $n$-dimensional space into $X$ is strongly approximable by closed embeddings. [10].

This theorem is due to Torunczyk and all the ingredients for its proof appear in

Recall that a $Z$-set $F$ in an ANR $X$ is a closed subset of $X$ such that each map of the Hilbert cube into $X$ is arbitrarily closely approximable by maps into $X-F$. A $\sigma$-Z-set in $X$ is a countable union of $Z$-sets in $X$.

THEOREM 2.6. Let $F$ be a dense $\sigma-Z$-set in a locally compact separable ANR $X$ and let $n \in \mathbb{N} \cup\{\infty\}$. The following statements hold:

(i) $X-F$ satisfies the discrete $n$-cells property if and only if $F$ is proximately $\mathrm{LC}^{n-1}$ in $X$.

(ii) If $F$ is $\mathrm{LC}^{n-1}$ in $X$, then $X-F$ satisfies the discrete $n$-cells property. The reverse implication is false.

Discussion of Proof. The proof of (i) for $n$ finite combines techniques found in [5 and 2] and essentially is due to Curtis. We use only the forward implication of (i) in this paper in showing that the complement in $I^{\infty}$ of the fake boundary set $B_{n}$ does not satisfy the discrete $(n+1)$-cells property. The proof of (ii) for $n$ finite appears as Theorem 3.6 of [3] and also in [2]. The fact that the reverse implication is false follows from an example of van Mill [7] of a boundary set in the Hilbert cube containing no arcs. (ii) is used in this paper to show that the complement in $I^{\infty}$ of $B_{n}$ satisfies the discrete $n$-cells property.

The proof of (i) and (ii) in case $n=\infty$ follows from the finite case once it is proved that $X-F$ satisfies the discrete $k$-cells property for all $k<\infty$ if and only if $X-F$ satisfies the discrete approximation property. The proof of this fact appears in [4].

3. The examples. For each $i \in \mathbb{N}$, let $W_{i}=\prod_{j \neq i}\left[-1+2^{-i}, 1-2^{-i}\right]_{j} \times\{1\}_{i} \subset I^{\infty}$. $W_{i}$ is a "shrunken endface" in the $i$ th coordinate direction and is a $Z$-set in $I^{\infty}$. $B_{0}=\bigcup_{i \in N} W_{i}$ is the Anderson-Curtis-van Mill $\sigma$-Z-set of Example 1.2. Theorems 
3.1-3.5 of [1] show that $B_{0}$ possesses properties (i)-(iv) of Example 1.2 and Sierpinski's Theorem [8] shows that $B_{0}$ is not locally continuum-connected, and therefore not a boundary set. Observe that $I^{\infty}-B_{0}$ satisfies the discrete 0 -cells property since $I^{\infty}-B_{0}$ is nowhere locally compact, but $I^{\infty}-B_{0}$ does not satisfy the discrete 1-cells property.

Let $\bar{A}$ be a dendrite whose endpoints are dense in $\bar{A}$. Thus, $\bar{A}$ is a nondegenerate uniquely arcwise connected Peano space, equivalently, a compact 1-dimensional AR, whose endpoints form a dense $G_{\delta}$-subset of $\bar{A}$. Let $F_{0}$ be any dense $\sigma$-compact collection of endpoints of $\bar{A}$, and let $A$ denote the complement of $F_{0}$ in $\bar{A}$. For instance, $F_{0}$ can be a countable dense subset of the endpoints of $\bar{A}$. Let

$$
F_{1}=\left(F_{0} \times \bar{A}\right) \cup\left(\bar{A} \times F_{0}\right)=\bar{A}^{2}-A^{2}
$$

and, for each $n>1$, let

$$
F_{n}=\left(F_{n-1} \times \bar{A}\right) \cup\left(\overline{A^{n}} \times F_{0}\right)=\bar{A}^{n+1}-A^{n+1} .
$$

Observe that $F_{n}$ is a $\sigma$-compact $\sigma$-Z-set in $\bar{A}^{n+1}$ for each $n \in \mathbb{N} \cup\{0\}$. For each $n \in \mathbb{N}$, define $B_{n}$ to be the $\sigma-Z$-set $\left(B_{0} \times \overline{A^{n}}\right) \cup\left(I^{\infty} \times F_{n-1}\right)$ in $I^{\infty} \times \bar{A}^{n} \approx I^{\infty}$. Routine set arguments and induction reveal that

$$
B_{n+1}=\left(B_{n} \times \bar{A}\right) \cup\left(I^{\infty} \times \bar{A}^{n} \times F_{0}\right) \subset I^{\infty} \times{\overline{A^{n+1}}}^{\infty} I^{\infty}
$$

and

$$
\left(I^{\infty} \times \bar{A}^{n}\right)-B_{n}=\left(I^{\infty}-B_{0}\right) \times A^{n} .
$$

EXAMPLE 3.1. For each $n \in \mathbb{N} \cup\{0\}, B_{n}$ is a dense $\sigma$-Z-set in $I^{\infty}\left(\approx I^{\infty} \times \overline{A^{n}}\right)$ that is not a boundary set though it satisfies (i)-(iv) of Example 1.2 and, in addition, satisfies:

(v) $B_{n}$ is $\mathrm{LC}^{n-1}$ in $I^{\infty}$ for $n \neq 0$ and $I^{\infty}-B_{n}$ satisfies the discrete $n$-cells property;

(vi) $B_{n}$ is not proximately $\mathrm{LC}^{n}$ in $I^{\infty}$ and thus $I^{\infty}-B_{n}$ does not satisfy the discrete $(n+1)$-cells property.

It is easy to verify that each $B_{n}$ satisfies (iv), and Theorems 3.1-3.5 of [1] apply to verify that each $B_{n}$ satisfies (i)-(iii). We already have observed that $I^{\infty}-B_{0}$ satisfies the discrete 0 -cells property and the verification of (v) for $n>0$ appears below.

If $U$ and $V$ are connected and, therefore, path connected open subsets of $I^{\infty}$ and $\bar{A}$, respectively, then since $B_{0}$ is dense in $I^{\infty}$ and $F_{0}$ is dense in $\bar{A},(U \times V) \cap B_{1}$ is path connected. This ensures that $B_{1}$ is $\mathrm{LC}^{0}$ in $I^{\infty} \times \bar{A} \approx I^{\infty}$. The fact that $B_{n}$ is $\mathrm{LC}^{n-1}$ in $I^{\infty}$ for $n>1$ follows from

THEOREM 3.2. Let $F$ be a dense subset of a space $X$ and let $n \in \mathbb{N}$. If $F$ is $\mathrm{LC}^{n-1}$ in $X$ and $X$ is $\mathrm{LC}^{n}$, then $(F \times \bar{A}) \cup\left(X \times F_{0}\right)$ is $\mathrm{LC}^{n}$ in $X \times \bar{A}$.

The proof of this theorem appears in [3]. Applying Theorem 3.2 to $B_{n+1}=\left(B_{n} \times\right.$ $\bar{A}) \cup\left(I^{\infty} \times F_{0}\right)$, where we have identified $I^{\infty} \times \overline{A^{n}}$ with $I^{\infty}$, shows that, since $B_{1}$ is $\mathrm{LC}^{0}$ in $I^{\infty}$, then for each $n>1, B_{n}$ is $\mathrm{LC}^{n-1}$ in $I^{\infty}$. This, coupled with the fact that $B_{n}$ is a dense $\sigma$-Z-set in $I^{\infty}$, then implies, by Theorem 2.6(ii), that $I^{\infty}-B_{n}$ satisfies the discrete $n$-cells property. 
We have left only to show that (vi) holds, which in turn implies that each $B_{n}$ is not a boundary set. If $B_{n}$ is proximately LC ${ }^{n}$ in $I^{\infty}$, then a straightforward and standard argument reveals that $B_{n}$ satisfies the following homological property. $\tilde{H}_{n}$ denotes reduced singular homology.

Definition 3.3. A subset $B$ of $I^{\infty}$ satisfies $H(n)$ for some nonnegative integer $n$ if: for every $\varepsilon>0$ there exists a $\delta>0$ such that, for every compactum $S \subset B$ with $\operatorname{diam} S<\delta$, there exists a compactum $K \subset B$ containing $S$ with diam $\mathrm{K}<\varepsilon$ such that, for every neighborhood $N(K)$ of $K$ in $I^{\infty}$, there exists a neighborhood $N(S)$ of $S$ in $I^{\infty}$ contained in $N(K)$ such that the inclusion induced homomorphism $\tilde{H}_{n}(N(S)) \rightarrow \tilde{H}_{n}(N(K))$ is the zero homomorphism.

$B_{0}$ does not satisfy $H(0)$ since $H(0)$ implies that $B$ is locally continuum-connected. The next proposition is used to show that $B_{n}$ for $n>0$ does not satisfy $H(n)$, and this implies that $B_{n}$ is not proximately $\mathrm{LC}^{n}$ in $I^{\infty}$. We have introduced this homological property because of the resulting ease in working with products.

Proposition 3.4. Let $B \subset I^{\infty}$ and define $B^{\prime}=(B \times \bar{A}) \cup\left(I^{\infty} \times F_{0}\right) \subset I^{\infty} \times \bar{A} \approx$ $I^{\infty}$. For $n \in \mathbb{N} \cup\{0\}$, if $B^{\prime}$ satisfies $H(n+1)$ in $I^{\infty} \times \bar{A}$, then $B$ satisfies $H(n)$ in $I^{\infty}$.

Armed with Proposition 3.4 it is easy to show that $B_{n}$ does not satisfy $H(n)$. Indeed, since for each nonnegative integer $i, B_{i+1}=\left(B_{i} \times \bar{A}\right) \cup\left(I^{\infty} \times F_{0}\right)$, where we have identified $I^{\infty} \times \bar{A}^{i}$ with $I^{\infty}$, successive applications of Proposition 3.4 show that if $B_{n}$ satisfies $H(n)$ for some $n>0$, then $B_{i}$ satisfies $H(i)$ for $0 \leqslant i \leqslant n$ and, in particular, $B_{0}$ satisfies $H(0)$, a contradiction.

Proof. Let $\varepsilon>0$ and let $\delta$ be the delta that works in $H(n+1)$ for $\varepsilon$ and $B^{\prime} \subset I^{\infty} \times \bar{A}$. Choose a cutpoint $a \in \bar{A}$ and identify $I^{\infty}$ with $I^{\infty} \times\{a\} \subset I^{\infty} \times \bar{A}$, and let $S$ be a compact subset of $B$ of diameter less than $\delta / 2$. Choose a small arc $L$ in $\bar{A}$ containing $a$ in its interior and having endpoints $e_{1}$ and $e_{2}$ in $F_{0}$, and let $D$ be a compact contractible subset of $I^{\infty}$ containing $S$ of diameter less than $\delta / 2$. Assume that $L$ has small enough diameter that the compact subset $S^{\prime}=(S \times L) \cup(D \times$ $\left.\left\{e_{1}, e_{2}\right\}\right)$ of $B^{\prime}$ has diameter less than $\delta$, and let $K^{\prime} \subset B^{\prime}$ be the compact subset of diameter less than $\varepsilon$ that is promised in $H(n+1)$. Since $K^{\prime} \subset B^{\prime}$ and $a \notin F_{0}$, $K=K^{\prime} \cap I^{\infty}=K^{\prime} \cap\left(I^{\infty} \times\{a\}\right)$ is contained in $B$. Note that $S \subset K$ and $K$ has diameter less than $\varepsilon$.

Let $U$ be an arbitrary neighborhood of $K$ in $I^{\infty}$ and let $U^{\prime}$ be a neighborhood of $K^{\prime}$ given by $U^{\prime}=(U \times \bar{A}) \cup \bar{U}$, where $\bar{U}$ is an open subset of $I^{\infty} \times \bar{A}$ that contains $K^{\prime}-(U \times \bar{A})$ and misses $I^{\infty} \times\{a\}$. Write $\bar{A}=\overline{A_{1}} \cup \overline{A_{2}}$, where $\overline{A_{i}}$ is a closed subset of $\bar{A}$ containing $e_{i}$ for $i=1,2$ and $\overline{A_{1}} \cap \overline{A_{2}}=\{a\}$. For an arbitrary subset $Y$ of $I^{\infty} \times \bar{A}$, define $Y_{i}=Y \cap\left(I^{\infty} \times \overline{A_{i}}\right)$ for $i=1,2$ and note that $\left\{U_{1}^{\prime}, U_{2}^{\prime}\right\}$ is an excisive couple of subsets [9].

$H(n+1)$ promises a neighborhood $W$ of $S^{\prime}$ contained in $U^{\prime}$ so that $\tilde{H}_{n+1}(W) \rightarrow$ $\tilde{H}_{n+1}\left(U^{\prime}\right)$ is zero. For $i=1,2$, since $S_{i}^{\prime}$ is a contractible subset of $W_{i}$, there exists a contractible neighborhood $V_{i}^{\prime}$ of $S_{i}^{\prime}$ in $W_{i}$. Let $V^{\prime}=V_{1}^{\prime} \cup V_{2}^{\prime}$ and $V=V_{1}^{\prime} \cap V_{2}^{\prime}$ and note that $\left\{V_{1}^{\prime}, V_{2}^{\prime}\right\}$ is an excisive couple of subsets and, since $V^{\prime} \subset W, \tilde{H}_{n+1}\left(V^{\prime}\right) \rightarrow$ $\tilde{H}_{n+1}\left(U^{\prime}\right)$ is zero. Also, $V$ is a neighborhood of $S$ in $I^{\infty}$ contained in $U$. 
Since $\left\{U_{1}^{\prime}, U_{2}^{\prime}\right\}$ and $\left\{V_{1}^{\prime}, V_{2}^{\prime}\right\}$ are excisive couples of subsets, we have the following commutative diagram, the rows being appropriate Mayer-Vietoris sequences and therefore exact, and the columns being inclusion induced maps.

$$
\begin{array}{ccccc}
\tilde{H}_{n+1}\left(V^{\prime}\right) & \stackrel{\tau}{\rightarrow} & \tilde{H}_{n}(V) & \rightarrow & \tilde{H}_{n}\left(V_{1}^{\prime}\right) \oplus \tilde{H}_{n}\left(V_{2}^{\prime}\right) \\
j_{*} \downarrow & & & \downarrow i_{*} \\
\tilde{H}_{n+1}\left(U^{\prime}\right) & \rightarrow & & & \\
& & \tilde{H}_{n}(U) & &
\end{array}
$$

Since $V_{1}^{\prime}$ and $V_{2}^{\prime}$ are contractible, $\tau$ is an epimorphism and therefore, since $j_{*}=0, i_{*}$ must be the zero homomorphism. This completes the proof of the proposition.

\section{REFERENCES}

1. R. D. Anderson, D. W. Curtis and J. van Mill, A fake topological Hilbert space, Trans. Amer. Math. Soc. 272 (1982), 311-321.

2. P. L. Bowers, Applications of general position properties of dendrites to Hilbert space topology, $\mathrm{Ph} . \mathrm{D}$. Dissertation, Univ. of Tennessee, 1983.

3. __ General position properties satisfied by finite products of dendrites, Trans. Amer. Math. Soc. (to appear).

4. __ Discrete cells properties in the boundary set setting, Proc. Amer. Math. Soc. (to appear).

5. D. W. Curtis, Boundary sets in the Hilbert Cube, Topology Appl. (to appear).

6. , Preliminary report, boundary sets in the Hilbert cube and applications to hyperspaces, preprint.

7. J. van Mill, $A$ boundary set for the Hilbert cube containing no arcs, Fund. Math. 118 (1983), 93-102.

8. W. Sierpinski, Un théorème sur les continus, Tohoku Math. J. 13 (1918), 300-303.

9. E. H. Spanier, Algebraic topology, McGraw-Hill, New York, 1966.

10. H. Torunczyk, Characterizing Hilbert space topology, Fund. Math. 111 (1981), 247-262.

Department of Mathematics, University of Tennessee, Knoxville, Tennessee 37996

Current address: Department of Mathematics, The Florida State University, Tallahassee, Florida 32306 